\title{
Übersicht
}

Obere Extremität 2020 $15: 172-178$ https://doi.org/10.1007/s11678-020-00599-5 Received: 17 June 2020

Accepted: 11 August 2020

Published online: 3 September 2020

(c) The Author(s) 2020

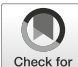

\author{
Alexandre Lädermann ${ }^{1,2,3}$ (D) Philippe Collin ${ }^{4}$ Patrick J. Denard ${ }^{5}$ \\ ' Division of Orthopaedics and Trauma Surgery, La Tour Hospital, Meyrin, Switzerland \\ ${ }^{2}$ Faculty of Medicine, University of Geneva, Geneva, Switzerland \\ ${ }^{3}$ Division of Orthopaedics and Trauma Surgery, Department of Surgery, Geneva University Hospitals, \\ Geneva, Switzerland \\ ${ }^{4}$ Centre Hospitalier Privé Saint-Grégoire (Vivalto Santé), Saint-Grégoire, France \\ ${ }^{5}$ Department of Orthopaedic \& Rehabilitation, Oregon Health \& Science University, Portland, USA
}

\section{Range of motion after reverse shoulder arthroplasty: which combinations of humeral stem and glenosphere work best?}

of different RSA designs and to provide recommendations accordingly.

\section{Glenoid configuration}

(RSA) design was excellent at restoring forward flexion, but had several designrelated complications including bony impingement and scapular notching [31, 38], instability [5], acromial fractures [19], limited range of motion (ROM) (particularly internal and external rotation; [20, 29]), and humeral stem loosening $[32,38]$. Many of these have been attributed to the initial Grammont design, which featured a medialized glenosphere and 155-degree straight stem (medial glenoid/medial humerus design) [12].

A variety of changes in prosthetic design have been proposed to address these issues either on the humeral side or on the glenoid side, the goal being to decrease scapular notching, maximize efficiency of the remaining rotator cuff, and improve stability as well as ROM. On the glenoid side, authors have promoted increased lateralization either with bone or metal $[4,15]$. On the humeral side, a more anatomic humeral inclination (i.e., 145 or 135 degrees) and inlay and onlay system designs have introduced a myriad of prosthetic configurations that has rendered analysis and delivery of universal guidelines difficult.

Therefore, the aim of this review was to evaluate the advantages and drawbacks
Glenoid configuration has an important effect on postoperative ROM. The three most important variables are glenoid offset, eccentricity, and glenosphere size. None of these latter parameters significantly influence the measured bone strains at the glenoid near the bone-implant interface [46].

\section{Glenoid offset (lateralization)}

The initial Grammont-style RSA utilized a glenosphere with a medialized center of rotation. While this design reliably improved forward elevation, the high rate of scapular notching and internal and external rotation deficit observed with this design have been attributed to the medialized glenoid design [12, 35]. To address these problems, glenoid lateralization has been proposed to decrease scapular notching, improve soft tissue tension, and increase impingement-free ROM. The glenoid component is considered as lateralized if lateralization equals or exceeds $5 \mathrm{~mm}$ compared to the Grammont design [50]. It is important to note that this lateralization of the center of rotation is relative to the implant designed by Grammont, but still medialized com- pared to the native glenohumeral joint. Lateralization can be achieved with either the placement of bone medial to the baseplate (BIO-RSA) [4] or with metallic lateralization via the baseplate or glenosphere. While both have been associated with clinical improvement [10,24], metallic lateralization appears to be potentially less subject to displacement, particularly with lateralization beyond $5 \mathrm{~mm}$ [8].

Basic scientific studies show several benefits of lateralization. In both sawbone [17] and computer models [15, 23, 33], lateralization improves ROM in all directions [33]. Lateralization also leads to improved stability [11].

The question remains as to how much lateralization is ideal. While clinical evidence is currently lacking, computer modeling suggests that $5-10 \mathrm{~mm}$ of lateralization relative to the native glenoid is ideal $[16,33,49]$. Nevertheless, clinical data to date have not necessarily proved that lateralization improves ROM [6] or outcome scores [21] compared to a traditional RSA. Greiner et al. performed a randomized controlled trial of 17 Grammont RSAs and 17 BIO-RSAs and reported no difference in Constant scores at 1 year postoperatively [14]. In a retrospective study, Athwal et al. did not observe substantial differences between Grammont RSA and BIO-RSA with respect to ROM, strength, or outcome 


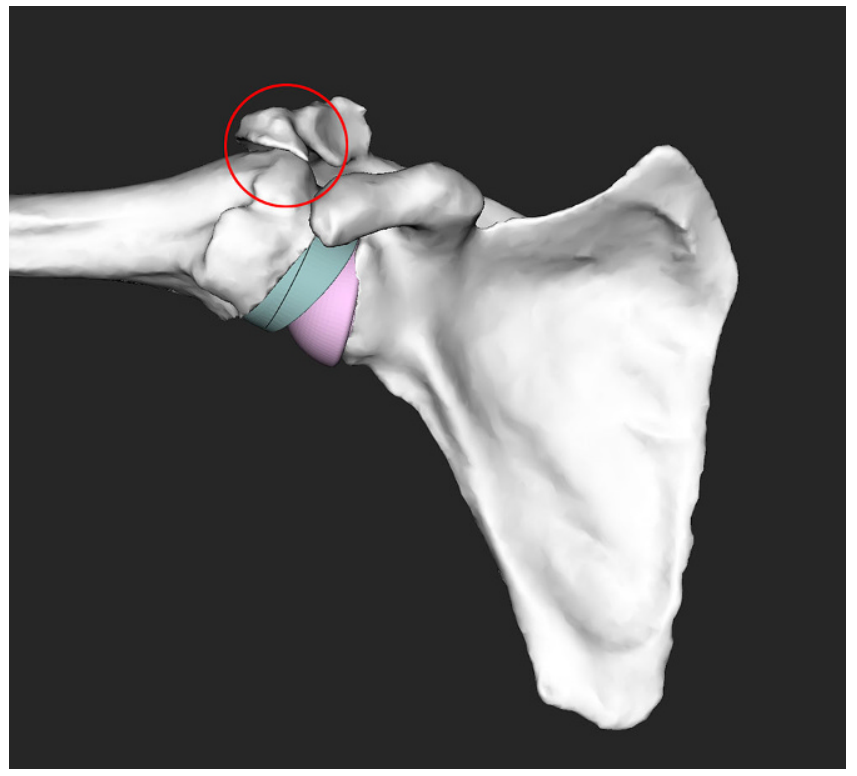

Fig. $1<$ Inexistent subacromial space prevents rotation in abduction in most configurations (from [31])

scores [1]. The frequency of scapular notching, however, was significantly higher $(P=0.022)$ in the RSA cohort than in the BIO-RSA cohort: $75 \%$ vs $40 \%$ [1]. This finding has also been reported by Zitkovsky et al. [51]. At 10 years followup, Kennon et al. confirmed that scapular notching rates are significantly higher with medialized components compared to lateralized ones $(77 \%$ in vs. $47 \%$, $P=0.013$ ) [22]. Notably, all of these studies utilized a 155-degree humeral prosthesis and thus further comparative studies are required with 135-degree and/or 145-degree protheses.

\section{Glenosphere eccentricity}

Inferior eccentric positioning of the glenosphere can also be used to decrease the adduction deficit and thus reduce scapular notching [23, 27]. Mizuno et al. previously reported that an inferiorly eccentric glenosphere reduced the severity of scapular notching with a 155-degree prosthesis [39], thereby improving postoperative rotations with elbow at side [31]. While the differences are small, the eccentric glenosphere provided the greatest ability to limit scapular notching while maximizing ROM by increasing the subacromial space $[27,33]$. Abduction is effectively positively correlated with acromiohumeral distance $(\mathrm{r}=0.93$; $p<0.001$ ), which is increased with an eccentric glenosphere [28]. Rotation in abduction is important in activities of daily living. Interestingly, the latter are impossible in most configurations due to inexistent subacromial space (• Fig. 1). Posteroinferior eccentricity can also improve extension and could additionally promote internal rotation hand in the back (- Fig. 2).

It is important to note, however, that inferior overhang of the glenosphere can be achieved either by an eccentric glenosphere or by a baseplate position. Conversely, an eccentric glenosphere with an improperly positioned superior baseplate will not provide clinical benefit. Thus, the surgeon must be cognizant of both the overhang of the given glenosphere relative to the selected baseplate, as well as any eccentricity in the glenosphere. Furthermore, the benefits of overhang or eccentricity must be weighed against the risks of nerve injury and acromial fracture associated with arm lengthening. The ideal amount of overhang relative to the glenoid appears to be about $2.5 \mathrm{~mm}$ based on clinical evidence [20].

Both theoretically and clinically, the size of the glenosphere influences postoperative ROM. Lädermann et al. found that a small glenosphere $(36 \mathrm{~mm})$ improves external rotation in abduction [27]. However, with the elbow at side, larger diameter glenospheres have been

\section{Glenosphere size}

shown to provide a greater impingementfree arc of motion and decrease scapular notching in biomechanical studies. Werner et al. reported superior values for extension and external rotation with a 39-mm glenosphere compared to a 36$\mathrm{mm}$ glenosphere [48]. Berhouet et al. demonstrated in a cadaveric study that a $42-\mathrm{mm}$ glenosphere was associated with improved rotational ROM compared to a $36-\mathrm{mm}$ glenosphere $(p<0.05)$ [3]. Another study comparing functional scores and ROM differences between two groups of patients, one receiving a 36$\mathrm{mm}$ glenosphere and the other receiving a 44-mm glenosphere, found that patients with the larger glenosphere had a 12-degree increase in external rotation in adduction compared to those with the smaller glenosphere $(p<0.001)$ [41]. Similarly, Mollon et al. showed that a $42-\mathrm{mm}$ glenosphere generated a $15-$ degree improvement in forward flexion and a 6-degree improvement in external rotation compared to the $38-\mathrm{mm}$ size, with an overall improvement in pain scores [40]. Finally, a study by Haidamous et al. demonstrated that larger glenosphere size and inferior positioning as well as posterior humeral offset are associated with improved postoperative ROM following RSA with a 135-degree humeral component [20]. Nevertheless, larger glenospheres limit abduction and rotations in abduction and are prone to higher volumetric wear rates and experienced greater polyethylene volume loss [18]. Additionally, one must consider patient size. Overstuffing can occur. Matsuki et al., for instance, demonstrated that small- and large-stature patients achieved lower improvement in ROM with an RSA system with only two glenosphere sizes (38 and 42), likely due to the fact that the small patients were overstuffed and the large patients did not have large enough glenospheres and/or lateralization [37].

\section{Humeral stem designs}

The primary humeral stem variables include stem geometry, neck-shaft angle, inlay versus onlay configuration, and humeral spacers. 


\section{Stem geometry}

Short curved stems were initially developed to facilitate implantation, maintain bone stock, and preserve rotator cuff insertion [25]. These stems also change humeral offset based on their positioning in the humeral canal. In one study, an onlay curve stem led to a $7-\mathrm{mm}$ increase in humeral offset compared with a traditional inlay straight Grammont prosthesis [26]. Curve stems decrease the acromiohumeral distance, which may lead to acromial impingement at small abduction angles [26]. On the other hand, humeral lateralization can be useful to compensate for medialization in the case of bone loss (- Fig. 3 ) and has been theorized to improve the mechanics of the remaining rotator cuff and deltoid musculature [45]. Stem design appeared to also have a substantial effect on abduction, as combinations with the straight Grammont stem had greater abduction (73-80\%) compared to those with the onlay curved stem (54-69\%; [28]).

\section{Neck-shaft angle (inclination)}

The Grammont RSA was designed as a non-anatomic implant with a relative valgus humeral neck inclination of 155 degrees. Based on the work by Gutierrez et al. [17], neck-shaft angle has decreased in modern prosthetic designs to a more varus or anatomic inclination of 145 or 135 degrees.

The neck-shaft angle is a major factor influencing length of the arm [30], but has little effect on humeral lateralization; by changing inclination from 155 degrees to 135 degrees within an onlay design, humeral offset only increased by about $2 \mathrm{~mm}$ [26].

Theoretically, compared to low neckshaft angle stems, higher inclinations (155 degrees) increased abduction by $100 \%$ and external rotation in abduction, regardless of glenosphere designs $[26,33]$. This finding is important as such external rotation is a major factor in the ability to perform activities of daily life such as hair care and facial grooming. However, a 155-degree inclination is associated with decreased adduction external rotation at the side

Obere Extremität 2020 · 15:172-178 https://doi.org/10.1007/s11678-020-00599-5

(c) The Author(s) 2020

A. Lädermann · P. Collin · P. J. Denard

\section{Range of motion after reverse shoulder arthroplasty: which combinations of humeral stem and glenosphere work best?}

\section{Abstract}

A variety of changes in prosthetic design have been proposed to address the complications of Grammont-style reverse shoulder arthroplasty (RSA). The Grammont RSA is excellent at restoring forward flexion, but often leads to little improvement in external and internal rotation. The purpose of this review was to analyze the effect of different glenoid and humeral configurations on range of motion (ROM) following RSA. With low neckshaft angle (135-145 degrees), glenosphere lateralization, and eccentricity, elevation remains unchanged, abduction slightly decreases, but a dramatic improvement in adduction, extension, and rotations with the elbow at the side are observed. However, all prosthetic designs should be considered on a case-by-case basis to optimize outcome.

Keywords

Shoulder pathology - Glenohumeral arthritis . Prosthesis design - Humeral and glenoid lateralization · Neck-shaft angle

\section{Bewegungsumfang nach inverser Schulterendoprothetik - welche Kombinationen von humeraler Komponente und Glenosphäre funktionieren am besten?}

\section{Zusammenfassung}

Mit vielfältigen Veränderungen im Prothesendesign wird versucht, den Komplikationen der inversen Schulterendoprothetik nach Grammont („reverse shoulder arthroplasty" [RSA]) entgegenzuwirken. Die GrammontRSA eignet sich ausgezeichnet für die Wiederherstellung der Vorwärtsflexion, bewirkt aber oft nur geringe Verbesserungen in der Außenund Innenrotation. Ziel der vorliegenden Übersicht war es, die Wirkung verschiedener glenoidaler und humeraler Konfigurationen auf den Bewegungsumfang nach RSA zu analysieren. Bei geringem Hals-Schaft-Winkel $\left(135-145^{\circ}\right)$, Glenosphärenlateralisierung und -exzentrizität bleibt die Elevation unverändert und die Abduktion ist leicht vermindert, es wird aber eine enorme Verbesserung in der Adduktion, Extension und Rotation mit seitlichem Ellbogen beobachtet. Zur Optimierung des Outcomes sollte jedoch jedes Prothesendesign mit Blick auf den individuellen Fall in Betracht gezogen werden.

\section{Schlüsselwörter}

Störungen des Schultergelenks · Glenohumerale Arthritis - Prothesendesign · Humerale und glenoidale Lateralisierung $\cdot$ Hals-SchaftWinkel
$[26,42,49]$ and extension due to medial bony impingement (which also leads to scapular notching) $[15,28,33,44,47$, 49]. Lateralization obtained via a lower neck-shaft angle increases adduction by $357 \%$ between a 155 -degree prosthesis compared with a 135-degree prosthesis. Also, an increase in extension of $381 \%$ and external rotation elbow at side of $116 \%$ are observed with a 135 degree prosthesis [26]. Such findings are important as external rotation with the elbow at the side and extension led to friction between the scapular pillar and the polyethylene insert. Even if this friction phenomenon does not limit ROM, it likely contributes to progressive polyethylene wear and scapular notching [31]. However, reducing the neck-shaft angle can have some negative effects on RSA contact mechanics. The contact area is reduced by $29 \%$ for 155 degrees to 145 degrees and by $59 \%$ for 155 degrees to 135 degrees. Consequently, there is an increase in maximum contact stress of $71 \%$ for 155 degrees to 145 degrees and of $286 \%$ for 155 degrees to 135 degrees [34].

Gobezie et al. published the results of a randomized controlled trial comparing humeral inclination of 135 degrees to 155 degrees among patients undergoing RSA with a neutral glenosphere (no lateralization) and found no significant 

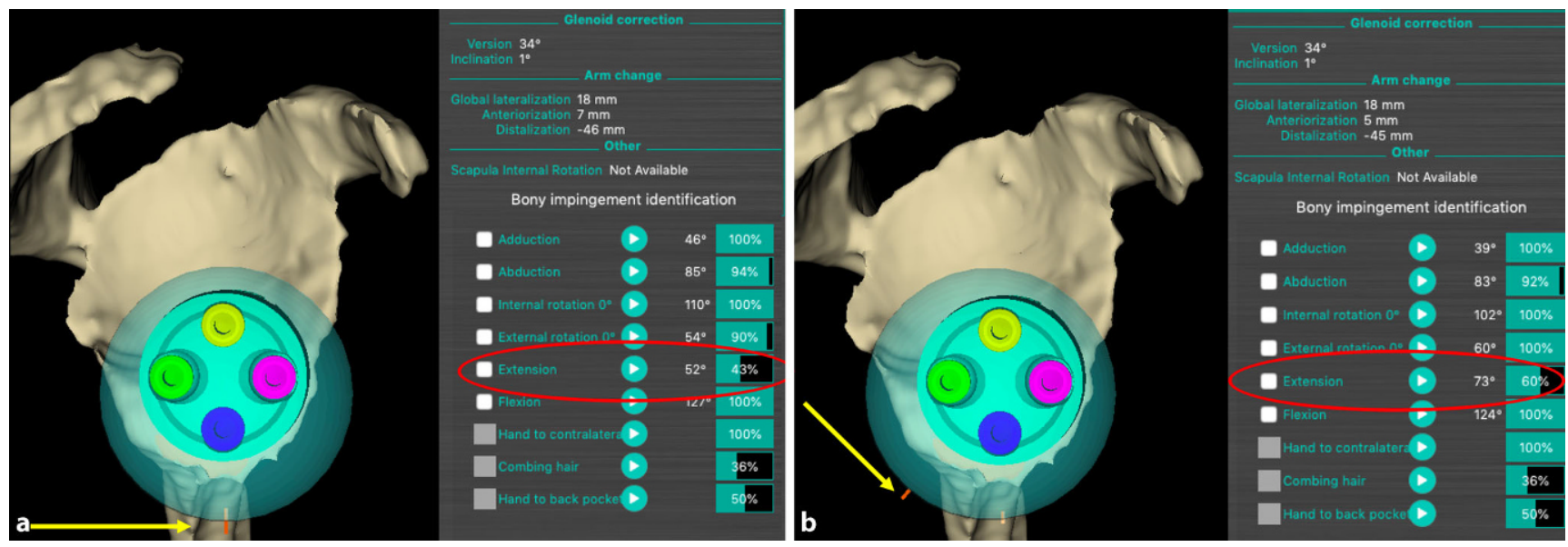

Fig. $2 \Delta$ Posteroinferior eccentricity can improve extension
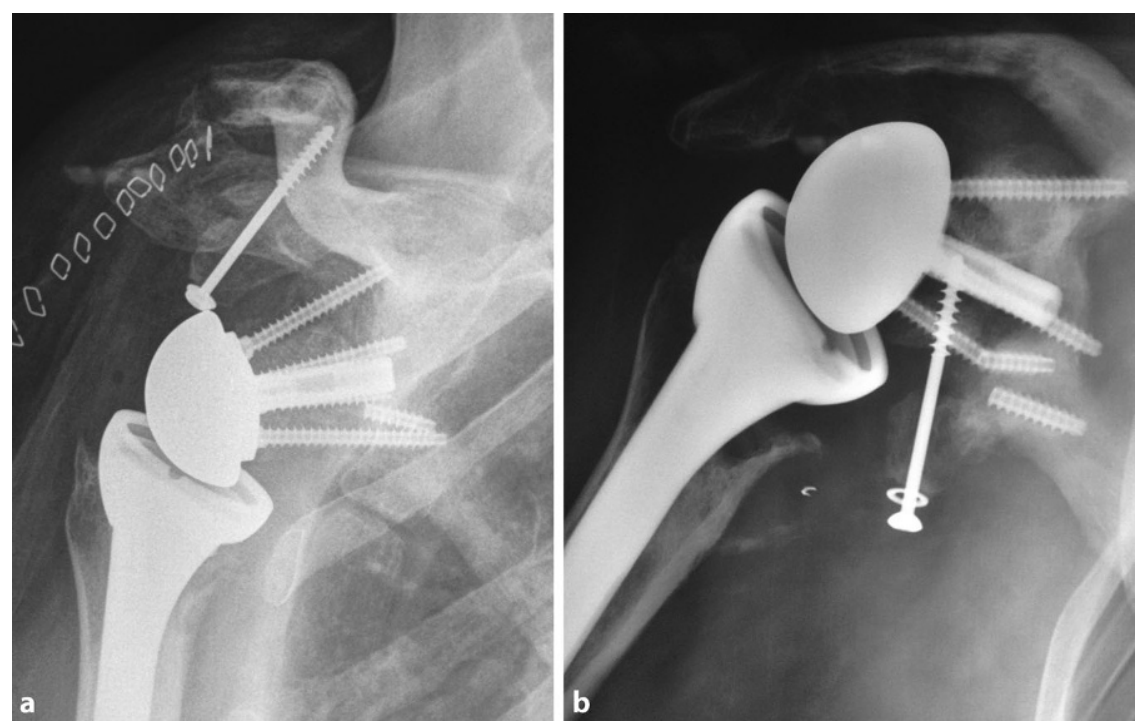

Fig. $3 \Delta$ Humeral lateralization can compensate for medialization in the case of bone loss

difference in forward flexion, external rotation, or functional outcomes [13]. They and other studies have confirmed that scapular notching is decreased with a 135-degree prosthesis $[9,13,51]$. A systematic review of 2222 shoulders comparing 135-degree and 155-degree prostheses reported higher rates of scapular notching in the 155-degree group (16.8\% vs. $2.8 \%$ ), improved external rotation in the 135-degree group, and no difference in instability of forward flexion between groups [9].

Lastly, in the case of fracture, RSA with a 135-degree neck-shaft angle is associated with higher tuberosity healing rates compared to 145 degrees or 155 degrees [43].

\section{Onlay vs. inlay}

Compared to inlay design, an onlay humeral design with the same 155degree inclination increased humeral offset by $6.6 \mathrm{~mm}$ [26]. Acromiohumeral distance varied by $9.8 \mathrm{~mm}$, with the smallest occurring with the onlay 135degree model and the largest occurring with a Grammont inlay 155 degrees. Compared to the inlay design, an onlay humeral design with the same 155-degree inclination decreased the acromioclavicular distance by $4.1 \mathrm{~mm}$. Compared to the onlay 155 degrees model, with the inlay 155 degrees model there was a 10 degree decrease ( 77.8 to 67.9 degrees) in abduction and a 5-degree (range, -15.3 to -20.2 degrees) increase in adduction [26].

Clinically, Beltrame et al. conducted a prospective clinical study comparing onlay and inlay steams. They found that an onlay design 145 degrees may provide better active external rotation, extension, and adduction [2]. However, there are numerous biases in their study (i.e., different neck-shaft angle and stem lateralization) preventing integration of their results in the present analysis.

A retrospective review reported a trend towards a higher rate of acromial fractures among patients with an onlay (12\%) as opposed to an inlay (4\%) system [36]. In a retrospective comparative radiological study, Haidamous et al. similarly showed that an onlay humeral stem design resulted in a 10$\mathrm{mm}$ increase in distalization compared to an inlay humeral stem, and a 2.5 times $(11.9 \%$ vs. $4.7 \%)$ increased risk of scapular spine fracture [19]. Thus, it seems that the combination of lateralization and distalization in an onlay system dramatically increases the incidence of scapular spine fractures.

Lengthening of the supraspinatus and infraspinatus is systematically observed with an onlay design. This is greatest using onlay stems $(7-30 \%)$ and lateralized glenospheres (13-31\%) [28]. Subscapularis lengthening is observed for onlay stems combined with lateralized glenospheres (5-9\%), while excessive subscapularis shortening is observed for the inlay stem combined with all gleno- 


\section{Übersicht}

Table 1 Implant design considerations to improve the range of motion (ROM) when performing a reverse shoulder arthroplasty

\section{Features/notes}

Glenoid

Offset (lateralization) Improve ROM and decreased notching by moving the center of rotation lateral to the glenoid

Eccentricity Improve ROM and decreased notching by distalization and prevention of impingement on the inferior glenoid

Glenosphere size Larger arc of motion

Humerus

Stem geometry

Neck shaft angle

(inclination)

Onlay vs. Inlay

Polyethylene insert

\section{Can lead to humeral lateralization}

135 Degrees leads to decreased notching, possibly improvement in external rotation, and improved tuberosity healing

Combined distalization and lateralization with onlay features increase risk of scapular spine fracture

Scapular notching limited by polyethylene notch spheres except the lateralized design $(>15 \%)$ [28].

\section{Polyethylene insert}

Since inferior impingement between the polyethylene and the scapula is systematic with the arm at the side, another potential way to limit friction and notching in external rotation is to create a notch in the polyethylene inferiorly between 3 and 9 oclock, as has been done in some prostheses (e.g., Arrow and SMR). Another solution to increase ROM is to reduce the depth of the polyethylene inlay. De Wilde et al. found that for every 3-mm decrease in depth of the polyethylene cup, ROM increased by 12 degrees [7].

\section{Discussion}

The literature is controversial with regard to possibilities of regaining ROM following RSA. While prosthetic designs vary and lead to substantial changes in computer models, many of the theoretical advantages have not been confirmed clinically. - Table 1 summarizes implant design considerations to improve ROM.

The results of this review reveal that the optimal compromise in ROM for a primary RSA without bone loss could be a lateral glenoid/medial (or intermediate) humerus design with a low neckshaft angle (145-135 degrees) and an inlay concept.

Glenoid and humeral prosthetic design needs to be chosen depending on pre- and intra-operative factors including, e.g., patient expectations, bone morphology, soft tissue status, such as rotator cuff or nerve, approaches, and surgical exposure. For example, it may not be possible to utilize a large glenosphere in all patients as they may not be appropriate for the anatomy of smaller individuals and might be more technically challenging. As a result, the surgeon must continuously weigh the benefits and possibilities of available implant-related variables regarding patients' specific conditions. The systematic use of patientspecific instrumentation and navigation as well as preoperative determination of components are obviously the next steps in providing more accurate component positioning and size, thereby improving ROM. Despite the advances made by glenoid lateralization and inferiorization, there remains ample opportunity for continued improvement and innovation in prosthetic design.

\section{Limitations}

The main limitation of this investigation is its non-systematic character. Another limitation is the omission of soft tissue tension, which can restrict ROM, particularly in revision or post-traumatic cases.

\section{Practical conclusion}

- Glenoid and humeral stem designs
change ROM and complication rates
after RSA.

- The optimal compromise in ROM for a primary RSA without bone loss might be a lateralized glenoid with a low neck-shaft angle (145-135 degrees) and an inlay concept.

- However, all prosthetic designs should be considered on a case-bycase basis to optimize outcome.

\section{Corresponding address}

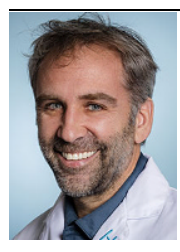

Alexandre Lädermann, PD-MD

Division of Orthopaedics and Trauma Surgery, La Tour Hospital

Av. J.-D. Maillard 3, 1217 Meyrin, Switzerland alexandre.laedermann@ gmail.com

Funding. Open access funding provided by University of Geneva

\section{Compliance with ethical guidelines}

Conflict of interest. A. Lädermann is a paid consultant for Wright, Arthrex and Medacta. He received Royalties from Wright. P. Collin is a paid consultant for Wright and Arthrex and received Royalties from Wright. P.J. Denard reports grants and personal fees from Arthrex

For this article no studies with human participants or animals were performed by any of the authors. All studies performed were in accordance with the ethical standards indicated in each case.

Open Access. This article is licensed under a Creative Commons Attribution 4.0 International License, which permits use, sharing, adaptation, distribution and reproduction in any medium or format, as long as you give appropriate credit to the original author(s) and the source, provide a link to the Creative Commons licence, and indicate if changes were made. The images or other third party material in this article are included in the article's Creative Commons licence, unless indicated otherwise in a credit line to the material. If material is not included in the article's Creative Commons licence and your intended use is not permitted by statutory regulation or exceeds the permitted use you will need to obtain permission directly from the copyright holder. To view a copy of this licence, visit http://creativecommons.org/licenses/by/4.0/.

\section{References}

1. Athwal GS, Macdermid JC, Reddy KM et al (2015) Does bony increased-offset reverse shoulder arthroplasty decrease scapular notching? J ShoulderElbow Surg 24:468-473

2. Beltrame A, Di Benedetto P, Cicuto C et al (2019) Onlay versus Inlay humeral steam in 
Reverse Shoulder Arthroplasty (RSA): clinical and biomechanical study. Acta Biomed 90:54-63

3. Berhouet J, Garaud P, Favard L (2014) Evaluation of the role of glenosphere design and humeral component retroversion in avoiding scapular notching during reverse shoulder arthroplasty. JShoulder Elbow Surg 23:151-158

4. Boileau P, Moineau G, Roussanne Y et al (2011) Bony increased-offset reversed shoulder arthroplasty: minimizing scapular impingement while maximizing glenoid fixation. Clin Orthop Relat Res 469:2558-2567

5. Chae J, Siljander M, Wiater JM (2018) Instability in reverse total shoulder arthroplasty. J Am Acad Orthop Surg 26:587-596

6. Collin P, Liu X, Denard PJ et al (2018) Standard versus bony increased-offset reverse shoulder arthroplasty: a retrospective comparative cohort study. JShoulderElbow Surg 27:59-64

7. De Wilde LF, Poncet $D$, Middernacht $B$ et al (2010) Prosthetic overhang is the most effective way to prevent scapular conflict in a reverse total shoulder prosthesis. Acta Orthop 81:719-726

8. Denard PJ, Lederman E, Parsons BO et al (2017) Finite element analysis of glenoid-sided lateralization in reverse shoulder arthroplasty. JOrthop Res 35:1548-1555

9. Erickson BJ, Frank RM, Harris JD et al (2015) The influence of humeral head inclination in reverse total shoulder arthroplasty: a systematic review. JShoulder Elbow Surg 24:988-993

10. Ernstbrunner L, Werthel JD, Wagner E et al (2017) Glenoid bone grafting in primary reverse total shoulder arthroplasty. J Shoulder Elbow Surg 26:1441-1447

11. Ferle M, Pastor MF, Hagenah J et al (2019) Effect of the humeral neck-shaft angle and glenosphere lateralization on stability of reverse shoulder arthroplasty: a cadaveric study. J Shoulder Elbow Surg 28:966-973

12. Gerber C, Pennington SD, Nyffeler RW (2009) Reverse total shoulder arthroplasty. J Am Acad Orthop Surg 17:284-295

13. Gobezie R, Shishani Y, Lederman E et al (2019) Can a functional difference be detected in reverse arthroplasty with 135 degrees versus 155 degrees prosthesis for the treatment of rotator cuff arthropathy: a prospective randomized study. JShoulder Elbow Surg 28:813-818

14. Greiner S, Schmidt C, Herrmann S et al (2015) Clinical performance of lateralized versus non-lateralized reverse shoulder arthroplasty: a prospective randomized study. J Shoulder Elbow Surg 24:1397-1404

15. Gutierrez S, Comiskey CA, Luo ZP et al (2008) Range of impingement-free abduction and adduction deficit after reverse shoulder arthroplasty. Hierarchy of surgical and implant-design-related factors. JBone Joint Surg Am 90:2606-2615

16. Gutierrez S, Greiwe RM, Frankle MA et al (2007) Biomechanical comparison of component position and hardware failure in the reverse shoulder prosthesis. J Shoulder Elbow Surg 16:S9-S12

17. Gutierrez S, Levy JC, Frankle MA et al (2008) Evaluation of abduction range of motion and avoidance of inferior scapular impingement in a reverse shoulder model. J Shoulder Elbow Surg 17:608-615

18. Haggart J, Newton MD, Hartner S et al (2017) Neer Award 2017: wear rates of 32-mm and 40-mm glenospheres in a reverse total shoulder arthroplasty wear simulation model. J Shoulder Elbow Surg 26:2029-2037
19. Haidamous $G$, Lädermann $A$, Frankle $M$ et al (2020) The risk of postoperative scapular spine fracture following reverse shoulder arthroplasty is increased with an onlay humeral stem. J Shoulder Elbow Surg. https://doi.org/10.1016/j.jse.2020.03. 036

20. Haidamous G, Lädermann A, Hartzler Ret al (2020) Radiographic parameters associated with excellent versus poor range of motion outcomes following reverse shoulder arthroplasty. Shoulder Elbow. https://doi.org/10.1177/1758573220936234

21. Helmkamp JK, Bullock GS, Amilo NR et al (2018) The clinical and radiographic impact of center of rotation lateralization in reverse shoulder arthroplasty: a systematic review. J Shoulder Elbow Surg 27:2099-2107

22. Kennon JC, Songy C, Bartels D et al (2020) Primary reverse shoulder arthroplasty: how did medialized and glenoid-based lateralized style prostheses compare at 10 years? J Shoulder Elbow Surg 29:S23-S31

23. Kim SJ, Jang SW, Jung KH et al (2019) Analysis of impingement-free range of motion of the glenohumeral joint after reverse total shoulder arthroplasty using three different implant models. JOrthop Sci 24:87-94

24. Klein SM, Dunning P, Mulieri $P$ et al (2010) Effects of acquired glenoid bone defects on surgical technique and clinical outcomes in reverse shoulder arthroplasty. J Bone Joint Surg Am 92:1144-1154

25. Lädermann A, Chiu JC, Cunningham G et al (2020) Do short stems influence the cervico-diaphyseal angle and the medullary filling after reverse shoulder arthroplasties? Orthop Traumatol Surg Res 106(2):241-246. https://doi.org/10.1016/j. otsr.2019.12.010

26. Lädermann A, Denard PJ, Boileau P et al (2015) Effect of humeral stem design on humeral position and range of motion in reverse shoulder arthroplasty. Int Orthop 39:2205-2213

27. Lädermann A, Denard PJ, Boileau P et al (2018) What is the best glenoid configuration in onlay reverse shoulder arthroplasty? Int Orthop 42:1339-1346

28. Lädermann A, Denard PJ, Collin P et al (2020) Effect of humeral stem and glenosphere designs on range of motion and muscle length in reverse shoulder arthroplasty. Int Orthop 44(3):519-530. https://doi.org/10.1007/s00264-019-04463-2

29. Lädermann A, Denard PJ, Tirefort J et al (2017) Subscapularis- and deltoid-sparing vs traditional deltopectoral approach in reverse shoulder arthroplasty: a prospective case-control study. JOrthop Surg Res 12:112

30. Lädermann A, Edwards TB, Walch G (2014) Arm lengthening after reverse shoulder arthroplasty: a review. Int Orthop 38:991-1000

31. Lädermann A, Gueorguiev B, Charbonnier C et al (2015) Scapular notching on kinematic simulated range of motion after reverse shoulde arthroplasty is not the result of impingement in adduction. Medicine 94:e1615

32. Lädermann A, Schwitzguebel AJ, Edwards TB et al (2019) Glenoid loosening and migration in reverse shoulder arthroplasty. Bone Joint J 101-B:461-469

33. Lädermann A, Tay E, Collin P et al (2019) Effect of critical shoulder angle, glenoid lateralization, and humeral inclination on range of movement in reverse shoulder arthroplasty. Bone Joint Res 8:378-386

34. Langohr GD, Willing R, Medley JB et al (2016) Contact mechanics of reverse total shoulder arthroplasty during abduction: the effect of neckshaft angle, humeral cup depth, and glenosphere diameter. JShoulder Elbow Surg 25:589-597

35. Lawrence C, Williams GR, Namdari S (2016) Influence of glenosphere design on outcomes and complications of reverse arthroplasty: a systematic review. Clin Orthop Surg 8:288-297

36. Leduc R, Salazar D, Garbis N (2019) Incidence of post-operative acromial fractures with onlay vs inlay reverse shoulder arthroplasty. J Shoulder Elbow Surg 28:e206

37. Matsuki K, King JJ, Wright TWetal (2018) Outcomes of reverse shoulder arthroplasty in small- and large-stature patients. J Shoulder Elbow Surg 27:808-815

38. Mélis B, Defranco M, Lädermann A et al (2011) An evaluation of the radiological changes around the Grammont reverse geometry shoulder arthroplasty after eight to 12 years. J Bone Joint Surg Br 93:1240-1246

39. Mizuno N, Denard PJ, Raiss P et al (2012) The clinical and radiographical results of reverse total shoulder arthroplasty with eccentric glenosphere. Int Orthop 36:1647-1653

40. Mollon B, Mahure SA, Roche CP et al (2016) Impact of glenosphere size on clinical outcomes after reverse total shoulder arthroplasty: an analysis of 297 shoulders. JShoulder Elbow Surg 25:763-771

41. Muller AM, Born M, Jung C et al (2018) Glenosphere size in reverse shoulder arthroplasty: is larger better for external rotation and abduction strength? J Shoulder Elbow Surg 27:44-52

42. Nelson R, Lowe JT, Lawler SM et al (2018) Lateralized center of rotation and lower neckshaft angle are associated with lower rates of scapularnotching and heterotopic ossification and improved pain for reverse shoulder arthroplasty at 1 year. Orthopedics 41:230-236

43. O'Sullivan J, Lädermann A, Parsons B et al (2020) A systematic review of tuberosity healing and outcomes following reverse shoulder arthroplasty for fracture according to humeral inclination of the prosthesis. JShoulderElbow Surg. https://doi.org/ 10.1016/j.jse.2020.03.032

44. Oh JH, Shin SJ, Mcgarry MH et al (2014)

Biomechanical effects of humeral neck-shaft angle and subscapularis integrity in reverse total shoulder arthroplasty. J Shoulder Elbow Surg 23:1091-1098

45. Parry S, Stachler S, Mahylis J (2020) Lateralization in reverse shoulder arthroplasty: a review. J Orthop 22:64-67

46. Pauzenberger L, Dwyer C, Obopilwe E et al (2019) Influence of glenosphere and baseplate parameters on Glenoid bone strains in reverse shoulder arthroplasty. BMC Musculoskelet Disord 20:587

47. Virani NA, Cabezas A, Gutierrez S et al (2013) Reverse shoulder arthroplasty components and surgical techniques that restore glenohumeral motion. JShoulder Elbow Surg 22:179-187

48. Werner BS, Chaoui J, Walch G (2018) Glenosphere design affects range of movement and risk of friction-type scapular impingement in reverse shoulder arthroplasty. Bone Joint J 100B:1182-1186

49. Werner BS, Chaoui J, Walch G (2017) The influence of humeral neck shaft angle and glenoid lateralization on range of motion in reverse shoulder arthroplasty. J Shoulder Elbow Surg 26:1726-1731

50. Werthel JD, Walch G, Vegehan E et al (2019) Lateralization in reverse shoulder arthroplasty: 


\section{Übersicht}

a descriptive analysis of different implants in current practice. Int Orthop 43:2349-2360

51. Zitkovsky HS, Carducci MP, Mahendraraj KA et al (2020) Lateralization and decreased neck-shaft angle reduces scapular notching and heterotopic ossification. J Am Acad Orthop Surg. https://doi. org/10.5435/jaaos-d-19-00808 